\title{
Absence of vertex correction for the spin Hall effect in p-type semiconductors
}

\author{
Shuichi Murakami ${ }^{1, \text { * }}$ \\ ${ }^{1}$ Department of Applied Physics, University of Tokyo, Hongo, Bunkyo-ku, Tokyo 113-8656, Japan
}

\begin{abstract}
We calculate an effect of spinless impurities on the spin Hall effect of the Luttinger model representing p-type semiconductors. The self-energy in the Born approximation becomes diagonal in the helicity basis and its value is independent of the wavenumber or helicity. The vertex correction in the ladder approximation vanishes identically, in sharp contrast with the Rashba model. This implies that in the clean limit the spin Hall conductivity reproduces the value of the intrinsic spin Hall conductivity calculated in earlier papers.
\end{abstract}

PACS numbers: 72.10.-d,72.20.-i,72.25.Dc

Understanding the dynamics of spins is a long-standing subject in semiconductor physics. Though there have been lots of experimental and theoretical studies toward this goal, there remain many obstacles to overcome before we can manipulate spins at our disposal in semiconductor spintronics devices1.2. One of the obstacles is efficient spin injection into semiconductors, and there have been many atempts for it. For example, spin injection from ferromagnetic metals has low efficiency, because of the band structure mismatch between semiconductors and metals, in particular by the conductivity mismatch ${ }^{3}$. On the other hand, while spin injection from ferromagnetic semiconductors such as $(\mathrm{Ga}, \mathrm{Mn}) \mathrm{As}^{\underline{4}}$ provides relatively high efficiency ${ }^{5}$, the Curie temperatures of such ferromagnetic semiconductors still remain lower than room temperature. Thus the spin injection, which constitutes the very first step for spintronic devices, is still under intensive investigation.

In such circumstances, recent theoretical proposals of an intrinsic spin Hall effect 6.7 have attracted much attention. In these works, it is theoretically predicted that an external electric field induces a dissipationless spin current in semiconductors. This intrinsic spin Hall effect is predicted for the Luttinger model representing bulk p-type semiconductors ${ }^{6}$, and for the Rashba model representing two-dimensional n-type semiconductors in heterostructure ${ }^{7}$. This intrinsic spin Hall effect is caused by the Berry phase ${ }^{8}$ in the momentum space. Berry-phase structure in momentum space is comprised in the electronic Bloch bands 9.10 .11 , and endows the carriers with an anomalous velocity. Due to the spin-orbit coupling, the carriers will then have spindependent trajectories in the presence of an external electric field, and it amounts to the intrinsic spin Hall effect. In bulk p-type semiconductors, the valence band has a spin splitting, giving rise to spin Hall effect by holedoping as proposed by the author and his collaborators ${ }^{6}$, whereas in bulk n-type semiconductors, the conduction band is doubly degenerate (if the Dresselhaus term is neglected), and the spin Hall effect does not emerge. Instead, by making the heterostructure from the n-type semiconductor, the Rashba spin splitting ${ }^{12.13}$ arises, and it gives rise to the spin Hall effect proposed by Sinova et $\mathrm{al}^{\underline{7}}$ Although an unambiguous experimental detec- tion remains to be done, many theoretical studies have appeared since the proposals of the intrinsic spin Hall effect 14.15.16.17.18.19.20.21.22.23.24.25.26. We note that these are different from an extrinsic spin Hall effect caused by spin-dependent scattering by impurities, which might be assumed as a main source of spin Hall effect in earlier papers $27,28,29$.

In order to argue possibilities of experimental detection, theoretical investigation of disorder effects is highly desired. In this paper, we mainly focus on the Luttinger model representing p-type semiconductors, $\stackrel{30}{=}$, and study disorder effects on the spin Hall effect. We shall also discuss on the Rashba model. For the Luttinger model, the effect of self-energy broadening was calculated by Schliemann and Loss ${ }^{17}$, and no other papers on disorder effect have appeared at present. In this paper, we study the effect of randomly distributed spinless impurities with short-ranged potential, by calculating both the self-energy correction in the Born approximation and the vertex correction in the ladder approximation. The calculation itself is analogous to that by Inoue et al. for the Rashba mode ${ }^{24.31}$. The resulting self-energy is of a simple form, independent of the wavevector $\mathbf{k}$. Remarkably, the vertex correction turns out to vanish identically, even away from the clean limit. Thus the spin Hall conductivity becomes identical with that of the intrinsic one calculated in ref. 6 . We note that it is in sharp contrast with the Rashba model, where the vertex correction completely kills the spin Hall effect within the Born and ladder approximations in the clean limit ${ }^{24.31}$.

The Luttinger Hamiltonian ${ }^{30}$ is written as

$$
H_{0}(\mathbf{k})=\frac{\hbar^{2}}{2 m}\left(\left(\gamma_{1}+\frac{5}{2} \gamma_{2}\right) k^{2}-2 \gamma_{2}(\mathbf{k} \cdot \mathbf{S})^{2}\right)
$$

where $\mathbf{S}=\left(S^{x}, S^{y}, S^{z}\right)$ are the spin-3/2 matrices, $\mathbf{k}=\left(k_{x}, k_{y}, k_{z}\right)$, and $k=|\mathbf{k}|$. For simplicity, we have put $\gamma_{2}=\gamma_{3}$ in the original Luttinger Hamiltonian. In this Hamiltonian, a helicity defined by $\lambda=\mathbf{k} \cdot \mathbf{S} / k$ is a good quantum number and can be used as a label for eigenstates. The helicity $\lambda$ can take values $\pm \frac{3}{2}, \pm \frac{1}{2} ; \lambda= \pm \frac{3}{2}$ correspond to the heavy-hole (HH) band and $\lambda= \pm \frac{1}{2}$ to the light-hole (LH) band. Thus the energy $E_{\mathbf{k} \lambda}$ of the eigenstatestate with wavenumber $\mathbf{k}$ and helicity $\lambda$ is 
expressed as

$$
\begin{aligned}
& E_{k, \pm \frac{3}{2}}=E_{k H}=\frac{\gamma_{1}-2 \gamma_{2}}{2 m} \hbar^{2} k^{2} \\
& E_{k, \pm \frac{1}{2}}=E_{k L}=\frac{\gamma_{1}+2 \gamma_{2}}{2 m} \hbar^{2} k^{2}
\end{aligned}
$$

which only depends on $k=|\mathbf{k}|$. By a unitary transformation $U_{\mathbf{k}}=e^{i \theta S_{y}} e^{i \phi S_{z}}$, where $\theta, \phi$ are spherical coordinates of $\mathbf{k},(\hat{k} \cdot \mathbf{S})$ is diagonalized as

$$
U_{\mathbf{k}}(\hat{k} \cdot \mathbf{S}) U_{\mathbf{k}}^{\dagger}=S_{z}=\operatorname{diag}\left(\frac{3}{2}, \frac{1}{2},-\frac{1}{2},-\frac{3}{2}\right),
$$

where $\hat{k}=\mathbf{k} / k$, and the Hamiltonian is diagonalized as well;

$$
U_{\mathbf{k}} H_{0} U_{\mathbf{k}}^{\dagger}=\operatorname{diag}\left(E_{k H}, E_{k L}, E_{k L}, E_{k H}\right) .
$$

Therefore, an eigenstate $|\mathbf{k} \lambda\rangle$ of the Hamiltonian $H$ with helicity $\lambda$ is expressed as

$$
|\mathbf{k} \lambda\rangle=U_{\mathbf{k}}^{\dagger}|\lambda\rangle
$$

where $|\lambda\rangle$ denote the eigenstate of $S_{z}$ with the eigenvalue $\lambda$.

We consider randomly distributed nonmagnetic impuries with short-ranged potential:

$$
V(\mathbf{r})=V \sum_{i} \delta\left(\mathbf{r}-\mathbf{R}_{i}\right)
$$

We shall calculate the Green function $G=(z-H)^{-1}$, $H=H_{0}+V$ in the self-consistent Born approximation from the unperturbed Green function $G^{(0)}=\left(z-H_{0}\right)^{-1}$. $G^{(0)}$ is diagonal in the helicity basis, with elements $g_{k \lambda}^{(0)}(z)=\left(z-E_{k \lambda}\right)^{-1}$. By taking the average $\langle\cdots\rangle_{\mathrm{AV}}$ over the impurity distribution, the lowest-order term of the self-energy in the Born approximation is written as

$$
\begin{aligned}
& \left\langle\left\langle\mathbf{k} \lambda\left|V G^{(0)} V\right| \mathbf{k}^{\prime \prime} \lambda^{\prime \prime}\right\rangle\right\rangle_{\mathrm{AV}} \\
& =\frac{n V^{2}}{L^{3}} \delta_{\mathbf{k} \mathbf{k}^{\prime \prime}} \sum_{\mathbf{k}^{\prime}, \lambda^{\prime}}\left\langle\mathbf{k} \lambda \mid \mathbf{k}^{\prime} \lambda^{\prime}\right\rangle\left\langle\mathbf{k}^{\prime} \lambda^{\prime} \mid \mathbf{k} \lambda^{\prime \prime}\right\rangle g_{k^{\prime} \lambda^{\prime}}^{(0)}
\end{aligned}
$$

where $n=N / L^{3}$ is a number density of the impurities.

We shall show that this self-energy (8) is written as $\Sigma \delta_{\mathbf{k k}^{\prime \prime}} \delta_{\lambda \lambda^{\prime \prime}}$, where $\Sigma$ is a constant. By substituting (6) into (8), we get

$$
\begin{aligned}
& \left\langle\left\langle\mathbf{k} \lambda\left|V G^{(0)} V\right| \mathbf{k}^{\prime \prime} \lambda^{\prime \prime}\right\rangle\right\rangle_{\mathrm{AV}} \\
& =\frac{n V^{2}}{L^{3}} \delta_{\mathbf{k k}^{\prime \prime}} \sum_{\mathbf{k}^{\prime}, \lambda^{\prime}}\left\langle\lambda\left|U_{\mathbf{k}} U_{\mathbf{k}^{\prime}}^{\dagger}\right| \lambda^{\prime}\right\rangle g_{k^{\prime} \lambda^{\prime}}^{(0)}\left\langle\lambda^{\prime}\left|U_{\mathbf{k}^{\prime}} U_{\mathbf{k}}^{\dagger}\right| \lambda^{\prime \prime}\right\rangle
\end{aligned}
$$

We note that the Green function $g_{k^{\prime} \lambda^{\prime}}^{(0)}$ is written as $a_{k^{\prime}}^{(0)}+$ $b_{k^{\prime}}^{(0)}\left(S_{z}^{2}\right)_{\lambda^{\prime} \lambda^{\prime}}$, where $a_{k^{\prime}}^{(0)}+\frac{1}{4} b_{k^{\prime}}^{(0)}=\left(z-E_{k^{\prime} L}\right)^{-1}, a_{k^{\prime}}^{(0)}+$ $\frac{9}{4} b_{k^{\prime}}^{(0)}=\left(z-E_{k^{\prime} H}\right)^{-1}$. Here $a_{k^{\prime}}^{(0)}$ and $b_{k^{\prime}}^{(0)}$ depend only on $k^{\prime}=\left|\mathbf{k}^{\prime}\right|$ and not on the direction of $\mathbf{k}^{\prime}$. The self-energy is then rewritten as

$$
\begin{aligned}
& \left\langle\left\langle\mathbf{k} \lambda\left|V G^{(0)} V\right| \mathbf{k}^{\prime \prime} \lambda^{\prime \prime}\right\rangle\right\rangle_{\mathrm{AV}} \\
& =\frac{n V^{2}}{L^{3}} \delta_{\mathbf{k k}^{\prime \prime}} \sum_{\mathbf{k}^{\prime}}\left\langle\lambda\left|U_{\mathbf{k}} U_{\mathbf{k}^{\prime}}^{\dagger}\left(a_{k^{\prime}}^{(0)}+b_{k^{\prime}}^{(0)} S_{z}^{2}\right) U_{\mathbf{k}^{\prime}} U_{\mathbf{k}}^{\dagger}\right| \lambda^{\prime \prime}\right\rangle \\
& \left.=\frac{n V^{2}}{L^{3}} \delta_{\mathbf{k k}^{\prime \prime}} \sum_{\mathbf{k}^{\prime}}\left\langle\lambda\left|U_{\mathbf{k}}\left(a_{k^{\prime}}^{(0)}+b_{k^{\prime}}^{(0)}\left(\hat{k}^{\prime} \cdot \mathbf{S}\right)^{2}\right) U_{\mathbf{k}}^{\dagger}\right| \lambda^{\prime \prime}\right\rangle 10\right)
\end{aligned}
$$

Among various terms in $\left(\hat{k}^{\prime} \cdot \mathbf{S}\right)^{2}$, the contributions from cross-terms such as $\hat{k}_{x}^{\prime} \hat{k}_{y}^{\prime} S_{x} S_{y}$ vanish by the summation over $\hat{k}^{\prime}$. Likewise, in a term $\left(\hat{k}_{x}^{\prime}\right)^{2} S_{x}^{2}$, for example, $\left(\hat{k}_{x}^{\prime}\right)^{2}$ can be replaced by $\frac{1}{3}\left(\hat{k}^{\prime}\right)^{2}=\frac{1}{3}$. Hence, $\left(\hat{k}^{\prime} \cdot \mathbf{S}\right)^{2}$ can be replaced by $\frac{1}{3} \mathbf{S}^{2}=\frac{5}{4}$, and we get

$$
\begin{aligned}
& \left\langle\left\langle\mathbf{k} \lambda\left|V G^{(0)}(z) V\right| \mathbf{k}^{\prime \prime} \lambda^{\prime \prime}\right\rangle\right\rangle_{\mathrm{AV}} \\
& =\frac{n V^{2}}{L^{3}} \delta_{\mathbf{k k}^{\prime \prime}} \sum_{\mathbf{k}^{\prime}}\left\langle\lambda\left|U_{\mathbf{k}}\left(a_{k^{\prime}}^{(0)}+\frac{5}{4} b_{k^{\prime}}^{(0)}\right) U_{\mathbf{k}}^{\dagger}\right| \lambda^{\prime \prime}\right\rangle \\
& =\frac{n V^{2}}{2 L^{3}} \delta_{\mathbf{k k}^{\prime \prime}} \delta_{\lambda \lambda^{\prime \prime}} \sum_{\mathbf{k}^{\prime}}\left(\frac{1}{z-E_{k^{\prime} L}}+\frac{1}{z-E_{k^{\prime} H}}\right)
\end{aligned}
$$

By a similar argument, the full self-energy in the selfconsistent Born approximation is seen to be diagonal in $\mathbf{k}$ and $\lambda$ :

$$
\begin{aligned}
& \left\langle\left\langle\mathbf{k} \lambda|G| \mathbf{k}^{\prime \prime} \lambda^{\prime \prime}\right\rangle\right\rangle_{\mathrm{AV}}=\frac{1}{\left(g_{k \lambda}^{(0)}\right)^{-1}-\Sigma} \delta_{\mathbf{k k}^{\prime \prime}} \delta_{\lambda \lambda^{\prime \prime}} \\
& \quad=g_{k \lambda} \delta_{\mathbf{k k}^{\prime \prime}} \delta_{\lambda \lambda^{\prime \prime}}, \\
& g_{\mathbf{k} H}=g_{\mathbf{k}, \frac{3}{2}}=g_{\mathbf{k},-\frac{3}{2}}, \quad g_{\mathbf{k} L}=g_{\mathbf{k}, \frac{1}{2}}=g_{\mathbf{k},-\frac{1}{2}} \\
& \Sigma=\frac{n V^{2}}{2 L^{3}} \sum_{\mathbf{k}^{\prime}}\left(g_{k^{\prime} L}+g_{k^{\prime} H}\right)
\end{aligned}
$$

where $g_{k \lambda}$ is a full Green function in the self-consistent Born approximation. The self-energy has several interesting properties; (i) it is diagonal in $\mathbf{k}$ and $\lambda$, and (ii) it is independent of $\mathbf{k}$ and of $\lambda$. It guarantees an assumption in the paper by Schliemann and Loss 17 .

Let us calculate a vertex correction for a charge current vertex within the ladder approximation. The charge current operator $J_{x}$ is written as

$$
J_{x}=\frac{e}{\hbar} \frac{\partial H}{\partial k_{x}}=\frac{e \hbar}{m}\left\{\left(\gamma_{1}+\frac{5}{2} \gamma_{2}\right) k_{x}-\gamma_{2}\left\{S_{x}, \mathbf{k} \cdot \mathbf{S}\right\}\right\}
$$

where $\{$,$\} is the anticommutator. The lowest order term$ of the vertex correction for $J_{x}$ in the ladder approxima- 
tion is given by

$$
\begin{aligned}
& \left\langle\left\langle\mathbf{k} \lambda\left|V G(z) J_{x} G\left(z^{\prime}\right) V\right| \mathbf{k}^{\prime} \lambda^{\prime}\right\rangle\right\rangle_{\mathrm{AV}} \\
& =\frac{V^{2}}{L^{6}} \sum_{\mathbf{k}_{1}, \lambda_{1}} \sum_{\mathbf{k}_{2}, \lambda_{2}} \sum_{i}\left\langle e^{-i\left(\mathbf{k}-\mathbf{k}_{1}\right) \cdot \mathbf{R}_{i}} e^{i\left(\mathbf{k}^{\prime}-\mathbf{k}_{2}\right) \cdot \mathbf{k}_{i}}\right\rangle_{\mathrm{AV}} \\
& \times\left\langle\mathbf{k} \lambda \mid \mathbf{k}_{1} \lambda_{1}\right\rangle\left\langle\mathbf{k}_{2} \lambda_{2} \mid \mathbf{k}^{\prime} \lambda^{\prime}\right\rangle g_{k_{1} \lambda_{1}}(z) g_{k_{2} \lambda_{2}}\left(z^{\prime}\right)\left\langle\mathbf{k}_{1} \lambda_{1}\left|J_{x}\right| \mathbf{k}_{2} \lambda_{2}\right\rangle \\
& =\frac{n V^{2}}{L^{3}} \delta_{\mathbf{k k}^{\prime}} \sum_{\mathbf{k}_{1}, \lambda_{1}, \lambda_{2}}\left\langle\mathbf{k} \lambda \mid \mathbf{k}_{1} \lambda_{1}\right\rangle\left\langle\mathbf{k}_{1} \lambda_{2} \mid \mathbf{k} \lambda^{\prime}\right\rangle \\
& \quad \times g_{k_{1} \lambda_{1}}(z) g_{k_{1} \lambda_{2}}\left(z^{\prime}\right)\left\langle\mathbf{k}_{1} \lambda_{1}\left|J_{x}\right| \mathbf{k}_{1} \lambda_{2}\right\rangle
\end{aligned}
$$

From eq. (13), $g_{\mathbf{k} \lambda}$ can be written as $g_{\mathbf{k} \lambda}=a_{k}+b_{k}\left(S_{z}^{2}\right)_{\lambda \lambda}$. This relation is useful in the calculation of the vertex correction as demonstrated below.

$$
\begin{aligned}
& \left\langle\left\langle\mathbf{k} \lambda\left|V G(z) J_{x} G\left(z^{\prime}\right) V\right| \mathbf{k}^{\prime} \lambda\right\rangle\right\rangle_{\mathrm{AV}} \\
& =\frac{n V^{2}}{L^{3}} \delta_{\mathbf{k k}^{\prime}} \sum_{\mathbf{k}_{1}}\langle\lambda| U_{\mathbf{k}} U_{\mathbf{k}_{1}}^{\dagger}\left(a_{k_{1}}(z)+b_{k_{1}}(z) S_{z}^{2}\right) U_{\mathbf{k}_{1}} \\
& \times J_{x}\left(\mathbf{k}_{1}\right) U_{\mathbf{k}_{1}}^{\dagger}\left(a_{k_{1}}\left(z^{\prime}\right)+b_{k_{1}}\left(z^{\prime}\right) S_{z}^{2}\right) U_{\mathbf{k}_{1}} U_{\mathbf{k}}^{\dagger}\left|\lambda^{\prime}\right\rangle \\
& =\frac{n V^{2}}{L^{3}} \delta_{\mathbf{k k}^{\prime}} \sum_{\mathbf{k}_{1}}\langle\lambda| U_{\mathbf{k}}\left\{a_{k_{1}}+b_{k_{1}}\left(\hat{k}_{1} \cdot \mathbf{S}\right)^{2}\right\} \\
& \times J_{x}\left(\mathbf{k}_{1}\right)\left\{a_{k_{1}}+b_{k_{1}}\left(\hat{k}_{1} \cdot \mathbf{S}\right)^{2}\right\} U_{\mathbf{k}}^{\dagger}\left|\lambda^{\prime}\right\rangle .
\end{aligned}
$$

Because the summand is an odd function of $\mathbf{k}_{1}$, this lowest order term of the vertex correction in the ladder approximation vanishes identically. Therefore, higher order terms in the ladder approximation vanish as well, and thus we conclude that the vertex correction for the current $J_{x}$ in the ladder approximation vanishes. This cancellation is between the intermediate states with $\mathbf{k}_{1}$ and $-\mathbf{k}_{1}$, i.e., due to parity. It is similar to the familiar example of vanishing vertex correction for the Fermi gas with spinless isotropic impurities. When we turn to the calculation of the spin Hall conductivity, we have to take into account the vertex correction in the charge current only. Therefore, because of this vanishing vertex correction, the spin Hall conductivity in the clean case reproduces the intrinsic value, which is obtained from a clean system without impurities from the outset 6.15 .32 . In other words, the result by taking a limit $1 / \tau \rightarrow 0$ before $\omega \rightarrow 0$ is identical with that by the reverse order of limits. The Luttinger model is free from a problem of order of limits.

By inspection, we can generalize the above discussion; for any inversion-symmetric models with $H(\mathbf{k})=$ $H(-\mathbf{k})$, the vertex correction vanish identically for shortranged scatterers. For example, even if we introduce the anisotropy in Luttinger parameters $\gamma_{2} \neq \gamma_{3}$, the vertex correction remains zero. If the impurity potential is longranged, forward scattering is preferred and the vertex correction no longer vanishes. A simple calculation similar to that for short-ranged scatterers shows that the resulting spin Hall effect is enhanced by a factor of $\tau_{\mathrm{tr}}^{\mathrm{SH}} / \tau$, where $\tau_{\mathrm{t} r}^{\mathrm{SH}}$ represents an effective transport lifetime for the spin Hall effect.
This is to be contrasted with the Rashba Hamiltonian studied by Inoue et al ${ }^{24.31}$ They studied the vertex correction within the ladder approximation for randomly distributed nonmagnetic impurities with isotropic potential. The result is remarkable; with the vertex correction, the spin Hall effect becomes zero in the clean limit 24.31 .33 , instead of $e / 8 \pi$, the universal value without impurities ${ }^{7}$. In their paper, they calculated the charge-current vertex appearing in the correlation function between the charge current and the spin current in the Kubo formalism. In the clean limit, the vertex correction for the charge current in the ladder approximation is -1 times the spin-dependent part of the charge current operator; therefore, the total charge-current vertex becomes spinindependent, yielding a vanishing spin Hall conductivity. This vanishing result by Inoue et al. corresponds to the order of limits, $\omega \rightarrow 0$ before $1 / \tau \rightarrow 0$, while the calculation of the intrinsic universal value by Sinova et al. corresponds to $1 / \tau \rightarrow 0$ before $\omega \rightarrow 0$. The former and the latter limits correspond to $L>l$ and $L<l$, respectively, where $L$ is the system size and $l$ is the mean free path. Nonetheless, it is not the end of the story. Two numerical calculations including disorder, one based on Kubo formula ${ }^{34}$ and the other on scattering theory ${ }^{26}$, indicate that the spin Hall effect in the clean limit remains the universal value $e / 8 \pi$. It contradicts the analytical result, and we do not have any answer to it. Here, we also note that Burkov et al ${ }^{22}$ formulated a theory of spincharge coupled transport, and discuss that the spin Hall conductivity vanishes in a diffusive regime. Thus, a comprehensive understanding of disorder effects for various strength of disorder is still to be desired.

Though the Rashba model is different from the Luttinger model, our calculation on the Luttinger model has some implications on the debate on the disorder effect in the Rashba model. To interpret the vanishing spin Hall conductivity in the clean limit ${ }^{24}$, Inoue et al. discuss that diffuse scattering efficiently scrambles the precession of spins such that no net spin Hall current remains. This picture is too simplified, since the spin Hall conductivity is not necessarily zero for general systems with spin-orbit coupling. Indeed, in the Luttinger model the vertex correction vanishes as we have seen. Furthermore, even for more general models with Rashba coupling, the spin Hall conductivity including the vertex correction is not necessarily zero in the clean limit. For example, instead of the simplest model of Rashba coupling

$$
H=\frac{\hbar^{2} k^{2}}{2 m}+\hbar \lambda\left(k_{y} \sigma_{x}-k_{x} \sigma_{y}\right),
$$

let us take a model

$$
H=\frac{\hbar^{2} k^{2}}{2 m}+\hbar\left(\lambda+\lambda_{1} k^{2}\right)\left(k_{y} \sigma_{x}-k_{x} \sigma_{y}\right)
$$

where $\lambda$ and $\lambda_{1}$ are constants. An extra term $\lambda_{1}$ is added here; this term should exist in general because it is allowed by symmetry. The vertex correction is calculated in the similar procedure as in refs. 24 31, and we can 
calculate the coefficient $\lambda^{\prime}$ for the vertex correction. After lengthy but straightforward calculation, we can see that the spin Hall conductivity is nonzero in the clean limit, even if the vertex correction is included within the ladder approximation. Thus, for the simplest model of Rashba coupling, (18), the complete cancellation of the spin Hall effect ${ }^{24,31}$ seems merely accidental, and not a consequence of any symmetries. This is also supported by the result with long-ranged scatterers, where the spin Hall conductivity including the vertex correction no longer vanishes 24 . To summarize, we can say that the spin Hall effect is not necessarily suppressed to zero by the vertex correction in general.
In conclusion, we consider an effect of spinless impurities on the spin Hall effect in the Luttinger model. We calculated the vertex correction for the charge current within the ladder approximation. For short-ranged scatters, the vertex correction is zero, and the spin Hall effect reproduces the intrinsic value, calculated previously from the system without impurities.

We thank G. E. W. Bauer, D. Culcer, J. Inoue, A. H. MacDonald, N. Nagaosa, Q. Niu, K. Nomura, J. Sinova, and S. -C. Zhang for fruitful discussions and helpful comments. This work is supported by Grant-in-Aids from the Ministry of Education, Culture, Sports, Science and Technology of Japan.
* Electronic address: murakami@appi.t.u-tokyo.ac.jp

1 S. A. Wolf, D. D. Awschalom, R. A. Buhrman, J. M. Daughton, S. von Molnár, M. L. Roukes, A. Y. Chtchelkanova, and D. M. Treger, Science 294, 1488 (2001).

2 D. D. Awschalom, N. Samarth, and D. Loss Semiconductor Spintronics and Quantum Computation (Springer-Verlag, Berlin, 2002).

3 G. Schmidt, D. Ferrand, L. W. Molenkamp, A. T. Filip, and B. J. van Wees, Phys. Rev. B 62, R4790 (2000).

${ }^{4}$ H. Ohno, Science 281, 951 (1998) and references therein.

${ }^{5}$ Y. Ohno, D. K. Young, B. Beschoten, F. Matsukura, H. Ohno, and D. D. Awschalom, Nature 402,790 (1999).

6 S. Murakami, N. Nagaosa, and S. C. Zhang, Science 301, 1348 (2003).

7 J. Sinova, D. Culcer, Q. Niu, N. A. Sinitsyn, T. Jungwirth, and A. H. MacDonald, Phys. Rev. Lett. 92, 126603 (2004).

8 M. V. Berry, Proc. R. Soc. London, Ser. A 392, 45 (1984).

9 Z. Fang, N. Nagaosa, K. S. Takahashi, A. Asamitsu, R. Mathieu, T. Ogasawara, H. Yamada, M. Kawasaki, Y. Tokura, and K. Terakura, Science 302, 92 (2003).

10 S. Murakami and N. Nagaosa, Phys. Rev. Lett. 90, 057002 (2003).

11 Y. Yao, L. Kleinman, A. H. MacDonald, J. Sinova, T. Jungwirth, D. S. Wang, E. Wang, and Q. Niu, Phys. Rev. Lett. 92, 037204 (2004)

12 E. I. Rashba, Sov. Phys. Solid State 2, 1109 (1960).

13 Y. A. Bychkov and E. I. Rashba, J. Phys. C 17, 6039 (1984).

14 D. Culcer, J. Sinova, N. A. Sinitsyn, T. Jungwirth, A. H. MacDonald, and Q. Niu, cond-mat/0309475

15 S. Murakami, N. Nagaosa and S. C. Zhang, cond-mat/0310005 to appear in Phys. Rev. B.

16 J. P. Hu, B. A. Bernevig, C. J. Wu, Int. J. Mod. Phys. B
17, 5991 (2003).

17 J. Schliemann and D. Loss, Phys. Rev. B 69, 165315 (2004)

18 N. A. Sinitsyn, E. M. Hankiewicz, W. Teizer and J. Sinova, cond-mat/0310315

19 S. Q. Shen, cond-mat/0310368

20 B. A. Bernevig, J. P. Hu, E. Mukamel, and S. C. Zhang, cond-mat/0311024

21 E. I. Rashba, Phys. Rev. B 68, 241315 (2003).

22 A. A. Burkov, A. S. Núñez, and A. H. MacDonald, cond-mat/0311328

23 L. Hu, J. Gao, S. Q. Shen, cond-mat/0401231

24 J. Inoue, G. E. W. Bauer, and L. W. Molenkamp, cond-mat/0402442

25 S. Q. Shen, M. Ma, X. C. Xie and F. C. Zhang, cond-mat/0403005

26 Y. Xiong and X. C. Xie, cond-mat/0403083

27 M. I. D'yakonov and V. I. Perel', ZhETF Pis. Red. 13, 657 (1971) [JETP Lett. 13, 467 (1971)].

28 J. E. Hirsch, Phys. Rev. Lett. 83, 1834 (1999).

29 S. Zhang, Phys. Rev. Lett. 85, 393 (2000).

30 J. M. Luttinger, Phys. Rev. 102, 1030 (1956).

31 J. Inoue, G. E. W. Bauer, and L. W. Molenkamp, Phys. Rev. B 67, 033104 (2003).

32 As discussed in ref. 15, definition of the spin current is not unique because the spin is not conserved. Nevertheless, whatever definition of the spin current one chooses, the vertex correction for the spin Hall conductivity vanishes.

33 R. Raimondi, M. Leadbeater, P. Schwab, E. Caroti and C. Castellani, Phys. Rev. B 64, 235110 (2001).

${ }^{34}$ K. Nomura, private communications. 